\title{
NECESSARY CONDITIONS FOR MAXIMAX PROBLEMS WITH APPLICATION TO AEROGLIDE OF HYPERVELOCITY VEHICLES $\dagger$
}

\author{
NGuyen X. Vinh and Ping Lu \\ Department of Aerospace Engineering, The University of Michigan, Ann Arbor, MI 48109-2140, U.S.A.
}

(Received 6 January 1987)

\begin{abstract}
This paper presents the necessary conditions for solving Chebyshev minimax (or maximax) problems with bounded control. The jump conditions obtained are applicable to problems with single or multiple maxima. By using Contensou domain of maneuverability, it is shown that when the maxima are isolated single points the control is generally continuous at the jump point in the minimax problems and discontinuous in the maximax problems in which the first time derivative of the maximax function contains the control variable. The theory is applied to the problem of maximizing the flight radius in a closed circuit glide of a hypervelocity vehicle and to a maximax optimal control problem in which the control appears explicitly with the first time derivative of the maximax function.
\end{abstract}

\section{INTRODUCTION}

In recent years, there is a strong interest in the so-called "Chebyshev minimax problem" in which the maximum of a scalar function $F(x)$ at an interior point is minimized[1,2]. Applications can be found in problems of reentry trajectories along which one seeks to minimize the peak deceleration or heating rate. A typical derivation of the necessary conditions based on the calculus of variations was given in[2] for one single maximum. This paper gives the necessary conditions for the minimax (or maximax) problems for the more general case where the control is bounded and the function $F(x)$ may have several equal maxima. In particular, we discuss the continuity of the control at the jump point where the function $F(x)$ reaches its maximum value. Several examples were considered to illustrate the application of the theory.

\section{NECESSARY CONDITIONS}

We consider the following problem

$$
\text { Minimize: (or Maximize) } \max _{t \in\left[t_{0}, t_{f}\right]} F(x)
$$

subject to

$$
\begin{aligned}
\dot{x} & =f(x, u), \quad x\left(t_{0}\right)=x_{0} \\
\omega\left(x_{f}\right) & =0
\end{aligned}
$$

$u \epsilon U$.

+Paper IAF-86-234 presented at the 37th Congress of the International Astronautical Federation, Innsbruck, Austria, 4-11 October 1986
In the equations $x$ is an $n$-dimensional state vector, $u$ is an $r$-dimensional control with $U$ being a bounded set in $R^{r}$. An admissible control $u$ is a piecewise continuous vector function with its value at $t, u(t) \epsilon U$. The eqn (3) defines an $m$-dimensional terminal manifold. Let $u_{j}$ be a scalar control. The minimax scalar function $F[x(t)]$ is said to be of order $q$ with respect to the control $u_{j}$ if $\mathrm{d}^{q} F / \mathrm{d} t^{q}$ is the derivative of $F$ which first contains $u_{j}$ explicitly.

To obtain the necessary conditions for the minimax problem we consider an augmented state $\bar{x}$ by adding to $x$ a new component $x_{n+1}$ such that

$$
\begin{aligned}
\dot{x} & =f(x, u) \\
\dot{x}_{n+1} & =0 \\
\omega\left(x_{f}\right) & =0 \\
x\left(t_{0}\right) & =x_{0}, \quad x_{n+1}\left(t_{0}\right)=\text { free. }
\end{aligned}
$$

The performance index to be minimized is

$$
J=x_{n+1}\left(t_{f}\right)
$$

with an artificially introduced state variable constraint such that

$$
g(\bar{x})=F(x)-x_{n+1} \leq 0 .
$$

In this Mayer type problem, we introduce an augmented adjoint variable $\bar{p}=\left(p, p_{n+1}\right)$ to form the Hamiltonian

$$
H=\bar{p}^{T} f=p^{T} f,
$$

with adjoint equations

$$
\dot{p}=-\frac{\partial H}{\partial x}, \quad \dot{p}_{n+1}=-\frac{\partial H}{\partial x_{n+1}}=0 .
$$

Since $x_{n+1}$ is obviously a constant, by the per- 
formance index (6), we minimize this quantity and by the inequality constraint (7) we indeed minimize the maximum value of $F(x)$ in the interval $\left[t_{0}, t_{f}\right]$. The problem is now cast into a well defined optimal control problem with state variable constraint where Pontryagin necessary conditions are available. At each instant, the Hamiltonian is maximized with respect to the control, and at the point where the boundary $g(\bar{x})=0$ is reached, we have the jump conditions

$$
\begin{aligned}
H^{+} & =H^{-} \\
\bar{p}^{+} & =\bar{p}^{-}+\mu \operatorname{grad}[g(\bar{x})],
\end{aligned}
$$

where $\mu$ is a constant parameter to be determined and subscripts ( - ) and $(+)$ denote the condition before and after the point of contact, respectively [3]. Pontryagin's derivation of the jump conditions aims at the case where after entering the boundary at the time $t_{1}$, the trajectory continues on that boundary for a time interval $\left(t_{1}, t_{1}^{\prime}\right)$. The proof requires that the components of the vector $\partial \dot{g} / \partial u$ are linearly independent. This implies that $q=1$ for all $u_{j}$. In this case, $F(x)$ may be allowed to display a flat maximum after $t_{1}$ (Fig. 1a). But this condition of linear independence is not enforced if the trajectory leaves the boundary immediately after the time $t_{1}$. This is the case where the maxima of $F(x)$ are isolated points (Fig. 1b). Then $q$ can be arbitrary in this case.

We first consider the case where the maxima at contact points are isolated points. They are necessarily equal in the present formulation.

At the time $t_{1}$, from the definition (7) of $g(\bar{x})$, we write the jump condition (11) explicitly

$$
\begin{aligned}
p^{+} & =p^{-}+\mu_{1} F_{x} \\
p_{n+1}^{+} & =p_{n+1}^{-}-\mu_{1} .
\end{aligned}
$$

Let $H^{*}$ be the maximized Hamiltonian. Then, at $t_{1}$

$$
H^{*}=p^{+^{r}} f^{+}=p^{-r} f^{-},
$$

where $f^{+}=f\left(x, u^{+}\right), f^{-}=f\left(x, u^{-}\right)$, with $u^{+}$and $u^{-}$ being the respective optimal control. Using the jump condition (12), we obtain

$$
p^{-r} f^{-}=p^{-r} f^{+}+\mu_{1} F_{x}^{T} f^{+} .
$$

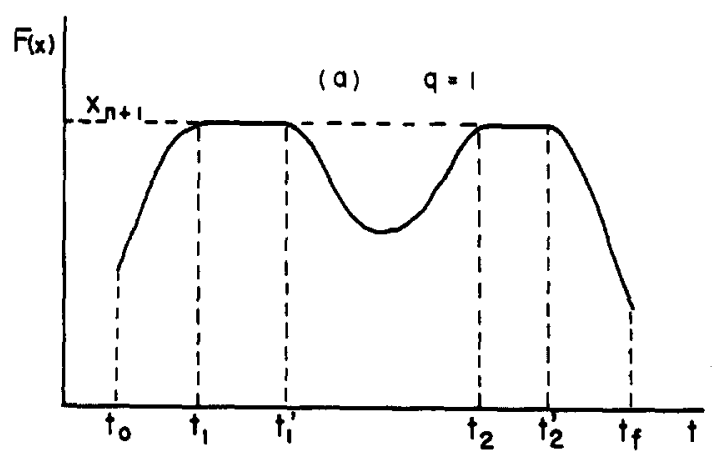

Rearranging the equation, we have

$$
p^{-T}\left(f^{-}-f^{+}\right)=\mu_{1} \dot{F}\left(x, u^{+}\right) .
$$

Similarly, we have the equation

$$
p^{+r}\left(f^{-}-f^{+}\right)=\mu_{1} \dot{F}\left(x, u^{-}\right) .
$$

In the case where $q>1, F$ does not contain $u$ explicitly and $\dot{F}(x)=0$ since $F(x)$ is a maximum at $t_{1}$. Then

$$
p^{+r}\left(f^{-}-f^{+}\right)=p^{-r}\left(f^{-}-f^{+}\right)=0 .
$$

It is enlightening to interpret this result using Contensou domain of maneuverability[4]. It is defined as the reachable domain $D(x)$ in the hodograph space

$$
V=\dot{x}=f(x, u) \text {, }
$$

for all $u \in U$. Then, the maximization of the Hamiltonian $H=p^{T} f$ leads to selecting the vector $V^{*}$ on the convex boundary of $D$. If the point is regular, that is if it is on the natural convex portion, $f\left(x, u^{+}\right)=f\left(x, u^{-}\right)$, and the control is continuous at the point $t_{1}$ (Fig. 2a). If the point is at the edge of a switching (Fig. 2b), the control is discontinuous[5]. The vector difference $\left(f^{-}-f^{+}\right)$is orthogonal to both $p^{-r}$ and $p^{+^{T}}$. In two-dimensional space, the three vector $p^{-}, p^{+}$and $F_{x}$ are collinear. Since the function $f(x, u)$ is known, it is possible to detect whether or not we have a possible switching by examining the domain of maneuverability. In general, we have the case of Fig. $2 \mathrm{a}$ and we can conclude that for the case where $q>1$ with isolated single or multiple maxima, although the adjoint variable is generally discontinuous, the control itself is continuous. For any order $q$, it will be shown that in general, $\mu_{1} \geq 0$ and, for $q=1$, in the minimax problem $\dot{F}\left(x, u^{-}\right) \geq 0$ and $\dot{F}\left(x, u^{+}\right) \leq 0$. Then, from eqn (15), since $p^{-T}\left(f^{-}-f^{+}\right)=H^{*}-p^{-r} f^{+} \geq 0$, we deduce that $\dot{F}\left(x, u^{+}\right) \geq 0$. Similarly, from eqn (16), we have $\dot{F}\left(x, u^{-}\right) \leq 0$. These relations lead to

$$
\dot{F}\left(x, u^{+}\right)=\dot{F}\left(x, u^{-}\right)=0 .
$$

We have the same conclusion for the continuity of the control as for the case where $q>1$. On the other hand in the maximax problem, the necessary condi-

Fig. 1. Multiple flat maxima and isolated maxima. 

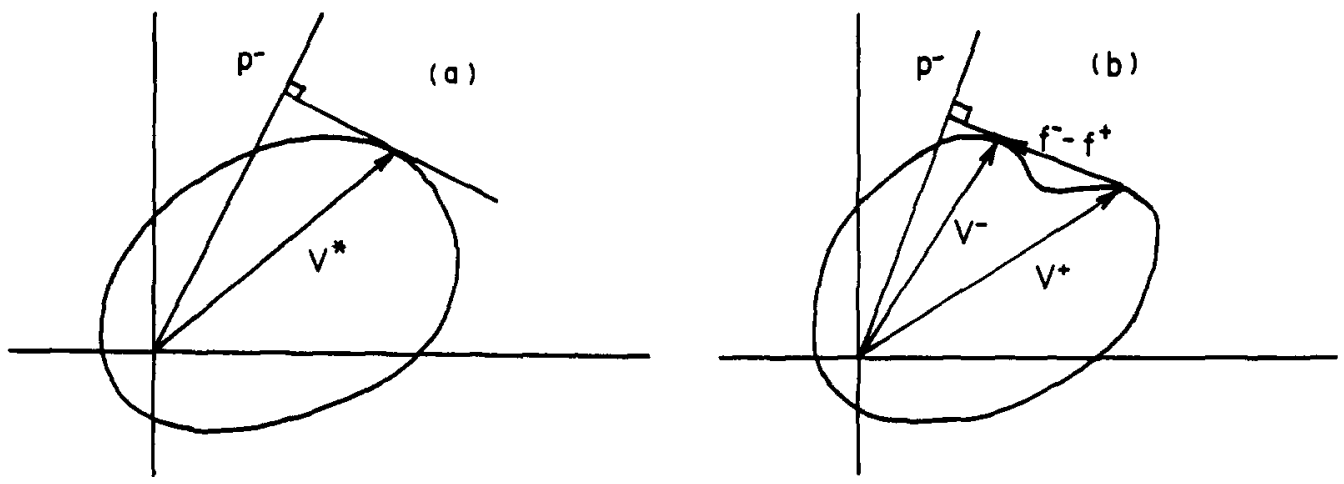

Fig. 2. Domain of maneuverability at jump point.

tions are the same, and in general, $\mu_{1}<0$. Then, we have $p^{-T}\left(f^{-}-f^{+}\right)>0$, and hence $\left(f^{-}-f^{+}\right) \neq 0$, and in general the control is discontinuous at the jump point.

In the minimax problem, and in the case of several maxima, since by eqn (9), $p_{n+1}=$ constant, and since $x_{n+1}\left(t_{0}\right)$ is free, $p_{n+1}^{-}\left(t_{1}\right)=0$. Then, from eqn (13), $p_{n+1}^{+}\left(t_{1}\right)=-\mu_{1}=p_{n+1}^{-}\left(t_{2}\right)$ since $p_{n+1}$ remains constant whether or not the trajectory stays on the boundary or leaves it immediately.

Again, we use the jump condition (13) at the entering point $t_{2}$ to obtain $p_{n+1}^{+}\left(t_{2}\right)=p_{n+1}^{-}\left(t_{2}\right)-\mu_{2}=$ $-\left(\mu_{1}+\mu_{2}\right)$. By repeating the process, we have finally

$$
\begin{aligned}
p_{n+1}^{+}\left(t_{k}\right)=-\left(\mu_{1}+\mu_{2}\right. & \left.+\ldots \mu_{k}\right) \\
& =p_{n+1}\left(t_{f}\right)=-\partial J / \partial x_{(n+1) f} .
\end{aligned}
$$

Therefore, for the minimax problem

$$
\mu_{1}+\mu_{2}+\ldots+\mu_{k}=1 \text {. }
$$

All the $\mu_{k}$ are non negative since $p_{n+1}(t)$ is a step function decreasing from 0 to -1 .

For the maximax problem, we simply observe that the jump conditions are local conditions at the jump points $t_{k}$. Then, we maximize the performance index (6), with the state constraint (7) changed into

$$
g(\bar{x})=x_{n+1}-F(x) \leq 0 .
$$

We have the same jump conditions (10) and (11), but now since we maximize $x_{n+1}\left(t_{f}\right), p_{n+1}\left(t_{f}\right)=$ $\partial J / \partial x_{(n+1) \mathcal{V}}=1$. All the $\mu_{k}$ are non positive and

$$
\mu_{1}+\mu_{2}+\ldots+\mu_{k}=-1 \text {. }
$$

Equations (20) and (22) are valid for the case of flat maxima where $q=1$ except that the $\mu_{k}$ can be negative or positive.

Finally, for the case where $q>1$ and $F(x)$ displays several flat maxima as shown in Fig. I(a), we can restrict to the case of one single scalar control and use Bryson and Ho's treatment of state inequality constraint of higher order[6]. The jump condition (11) is replaced by the condition at the entry point $t_{k}$

$$
\begin{aligned}
p^{+}\left(t_{k}\right) & =p^{-}\left(t_{k}\right)+\mu_{k} F_{x}+N_{x} \eta_{k} \\
p_{n+1}^{+}\left(t_{k}\right) & =p_{n+1}^{-}\left(t_{k}\right)-\mu_{k}
\end{aligned}
$$

where $N_{x}$ is an $n \times(q-1)$ matrix and $\eta_{k}$ is a $(q-1)$ column vector

$$
\begin{aligned}
N_{x} & =\left[\frac{\partial \dot{F}}{\partial x}, \frac{\partial \ddot{F}}{\partial x}, \ldots, \frac{\partial F^{(q-1)}}{\partial x}\right] \\
\eta_{k} & =\left[\eta_{1}^{k}, \eta_{2}^{k}, \ldots, \eta_{q-1}^{k}\right]^{T} .
\end{aligned}
$$

For the minimax problem, the relation (20) is still valid.

We shall apply the maximax theory to some problems of particular interest.

\section{MAXIMUM FLIGHT RADIUS IN CLOSED CIRCUIT GLIDE}

Consider the glide in a horizontal plane of a lifting vehicle with eqns [7]:

$$
\begin{aligned}
& \dot{x}=v \cos \psi, \quad \dot{y}=v \sin \psi \\
& \dot{v}=-\frac{v^{2}}{2 E^{*} \omega}\left[1+\frac{\omega^{2}}{v^{4}}\left(1+\tan ^{2} \sigma\right)\right], \quad \psi=\frac{\tan \sigma}{v}
\end{aligned}
$$

Here we use dimensionless variables with coordinates $x$ and $y$, speed $v$ and heading angle $\psi$. The single control is the bank angle $\sigma$, restricted to a maximum value $\sigma_{\max }=70^{\circ}$. In the equations, $E^{*}$ is the $\max$ imum lift-to-drag ratio of the vehicle taken as $E^{*}=20$ for a low altitude glider. The parameter $\omega$ includes other physical characteristics of the vehicle and is inversely proportional to the density of the atmosphere at the flight altitude. Hence we use it as defining the flight level with higher $\omega$ for higher altitude. The flight starts at the origin with the initial condition

$$
t_{0}=0, \quad x_{0}=y_{0}=\psi_{0}=0, \quad v_{0}=1 .
$$

It is proposed to modulate the bank angle to fly a closed circuit such that the flight range from the origin is maximized. Hence, we maximize the maximum of the function

$$
F=x^{2}+y^{2}
$$

in the interval $t \epsilon\left[t_{0}, t_{f}\right]$. The final condition imposed is

$t_{f}=$ free $, x_{f}=y_{f}=0, \psi_{f}=$ free, $v_{f}=\sqrt{\omega / \lambda_{\max }}$. 
The final speed $v_{f}$ is the stall speed attained when the normalized lift coefficient $\lambda$ attains its maximum value, taken as $\lambda_{\max }=2$.

First, we notice that

$$
\dot{F}(x, y)=2 v(x \cos \psi+y \sin \psi) \text {. }
$$

The derivative $\dot{F}$ does not contain the control, and we have $q>1$. It is easy to show that the domain of maneuverability in the $(\dot{\psi}, \dot{v})$ space is a parabola. Hence, it is convex with the only possible switching
Using the first two equations in the last equation, we deduce that $C_{3}^{+}=C_{3}^{-}$, and since $\psi_{f}$ is free, we obtain from $p_{\psi}\left(t_{f}\right)=0$

$$
C_{3}^{+}=C_{3}^{-}=0 \text {. }
$$

We see that the adjoint vector $p$ is discontinuous although the control in the bank angle is continuous. This can be verified directly by noticing that for a time free problem, $C_{0}=0$, and using the Hamiltonian integral with the solution for the adjoint variables and the control law (32) to obtain explicitly

$$
\tan \sigma=\frac{-v^{2}\left(C_{1} \cos \psi+C_{2} \sin \psi\right)+\sqrt{v^{4}\left(C_{1} \cos \psi+C_{2} \sin \psi\right)^{2}+\left(C_{1} y-C_{2} x\right)^{2}\left[\left(v^{4} / \omega^{2}\right)+1\right]}}{\left(C_{1} y-C_{2} x\right)}
$$

of the control between $\sigma_{\max }$ and $-\sigma_{\max }$. It would require that $p_{\psi}=0$. Hence, the control is continuous at jump point.

The Hamiltonian of the problem is

$$
\begin{aligned}
H=p_{x} v & \cos \psi+p_{y} v \sin \psi-p_{v} \frac{v^{2}}{2 E^{*} \omega} \\
\times & {\left[1+\frac{\omega^{2}}{v^{4}}\left(1+\tan ^{2} \sigma\right)\right]+p_{\psi} \frac{\tan \sigma}{v} . }
\end{aligned}
$$

The maximization of the Hamiltonian leads to the optimal bank angle

$$
\tan \sigma=\frac{p_{\psi}}{p_{v}}\left(\frac{E^{*} v}{\omega}\right)
$$

In this problem, we have the classical integrals: $p_{x}=C_{1}, \quad p_{y}=C_{2}, p_{\psi}=C_{1} y-C_{2} x+C_{3}, \quad H=C_{0}$.

Hence, the adjoint equations are completely integrated, and the optimal control is expressed explicitly in terms of the state variables and constants of integration.

The function $F$ has only one maximum in the interval $\left[t_{0}, t_{f}\right]$. Therefore, for a maximax problem, $\mu_{1}=-1$ and we can write the jump condition (11) at the point $\left(x_{1}, y_{1}\right)$ where $F$ is maximized.

$$
\begin{aligned}
C_{1}^{+} & =C_{1}^{-}-2 x_{1} \\
C_{2}^{+} & =C_{2}^{-}-2 y_{1} \\
C_{1}^{+} y_{1}-C_{2}^{+} x_{1}+C_{3}^{+} & =C_{1}^{-} y_{1}-C_{2}^{-} x_{1}+C_{3}^{-} .
\end{aligned}
$$

The control is used with $C_{1}^{-}$and $C_{2}^{-}$before the jump point $t_{1}$ and $C_{1}^{+}$and $C_{2}^{+}$after the jump while using eqn (34) for the transformation of the constants. The time $t_{1}$ is obtained using the condition

$$
\dot{F}\left(t_{1}\right)=2 v_{1}\left(x_{1} \cos \psi_{1}+y_{1} \sin \psi_{1}\right)=0 .
$$

The fact that the control is continuous at the jump point is seen by using eqns (34) and (37) to verify that

$$
\begin{aligned}
C_{1}^{+} \cos \psi_{1}+C_{2}^{+} \sin \psi_{1} & =C_{1}^{-} \cos \psi_{1}+C_{2}^{-} \sin \psi_{1} \\
C_{1}^{+} y_{1}-C_{2}^{+} x_{1} & =C_{1}^{-} y_{1}-C_{2}^{-} x_{1} .
\end{aligned}
$$

We also notice that since $F$ does not contain $v$ and $\psi$, $p_{v}$ and $p_{\psi}$ are continuous. Therefore, the bank angle is continuous in virtue of eqn (32). The computation of the optimal trajectory consists of selecting the initial constants $C_{1}^{-}$and $C_{2}^{-}$for the integration, with transformation at the jump point, until the stall speed $v_{f}$ while using the conditions $x_{f}=y_{f}=0$ for adjustment. Several trajectories with various values of $\omega$ are shown in Fig. 3. It is seen that there exists an altitude, in terms of the parameter $\omega$, providing the largest maximum range.

We have also considered the problem of minimum flight radius. The difference is that since it is a minimax problem, $\mu_{1}=1$. We have plotted in Fig. 4 , at the flight level $\omega=0.74$, the two trajectories for maximum radius, with $R_{\max }=1.83$, and minimum radius, with $R_{\min }=0.596$ using the same terminal conditions. The bank controls for the two trajectories are markedly different as shown in Fig. 5.

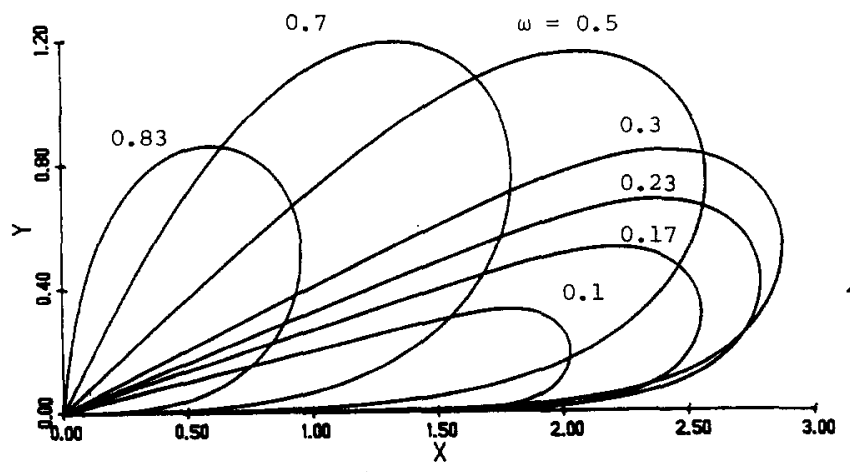

Fig. 3. Optimal trajectories for maximum fight radius at several flight altitudes. 


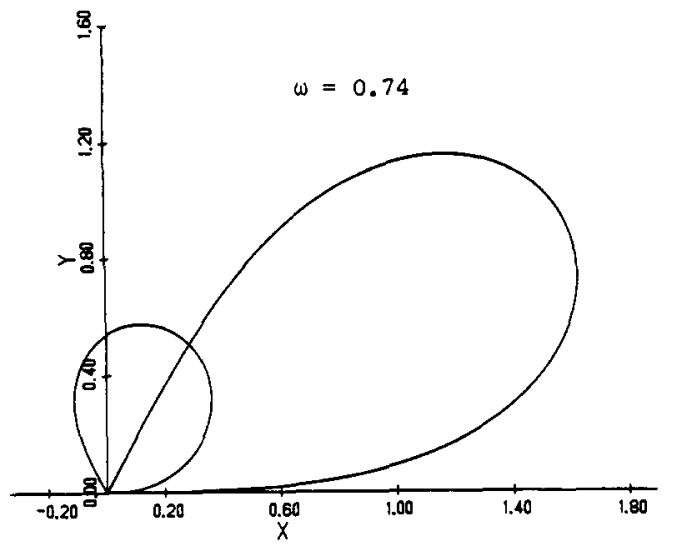

Fig. 4. Optimal trajectories with maximum and minimum radius.

Although both bank angles start and end at zero value, in the maximax problem the bank increases slowly to its peak value at the jump point and decreases while, in the minimax problem the bank angle increases rapidly to its maximum allowable value of $70^{\circ}$ and stays at this boundary for a while for a tight turning before decreasing to zero. By taking the derivative of eqn (32), it is a simple exercise to show that $\dot{\sigma}$ is continuous except at the jump point where

$$
\frac{\dot{\sigma}^{-}-\dot{\sigma}^{+}}{\sin 2 \sigma}=\frac{ \pm v_{1} \sqrt{x_{1}^{2}+y_{1}^{2}}}{p_{\psi}}=\frac{ \pm R v_{1}}{p_{\psi}}
$$

where the + sign on the righthand side corresponds to the maximax problem and the - sign for the minimax problem. Therefore, the variation of the optimal bank angle has a discontinuity in the slope at the jump point except when it is on the boundary $\sigma_{\max }$.

The example considered is a test example using a flat earth model with a vehicle having high lift performance. We now consider the glide at very high altitude over a spherical earth of a hypervelocity vehicle. By restricting the flight to constant altitude,

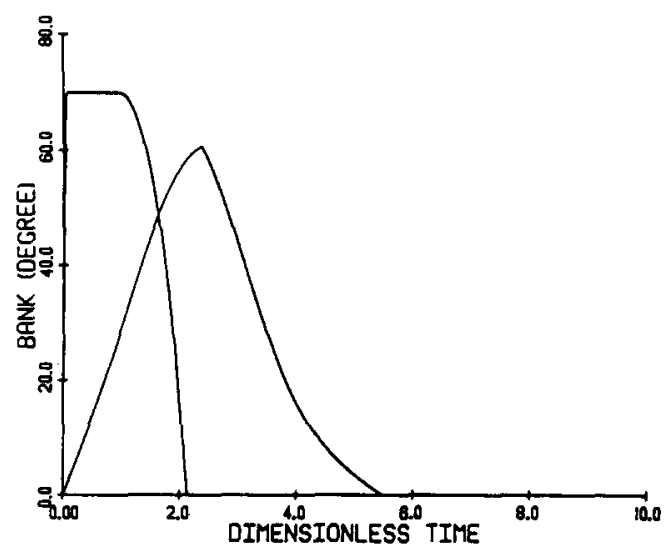

Fig. 5. Bank control for maximum and minimum radius. we have the dimensionless equations of motion[8]:

$$
\begin{aligned}
\dot{\theta} & =\cos \psi / \cos \phi, \quad \phi=\sin \psi \\
\dot{v} & =-\frac{v}{E^{*} \omega}\left[1+\frac{\omega^{2}(1-v)^{2}}{v^{2}}\left(1+\tan ^{2} \sigma\right)\right] \\
\dot{\psi} & =\frac{(1-v)}{v} \tan \sigma-\cos \psi \tan \phi .
\end{aligned}
$$

Here, $\theta$ denotes the longitude of the vehicle, $\phi$ its latitude, and $\psi$ is the heading. The control is the bank angle $\sigma$. Again, $E^{*}$ is the maximum lift-to-drag ratio of the vehicle taken as $E^{*}=7$ for the computation. As for the case of a flat earth, the constant $\omega$ is a parameter specifying the flight altitude. More specifically, we define

$$
\omega=\frac{2 m}{\rho S C_{L}^{*} r},
$$

where $\rho$ is the density at the flight distances $r, m$ the mass of the vehicle with reference area $S$ and $C_{L}^{*}$ is the lift coefficient at maximum lift-to-drag ratio. The speed variable $v$ is defined as the square of the ratio of the actual speed to the circular speed at distance $r$, and the independent variable is the dimensionless arc length $s$, that is

$$
v=\frac{V^{2}}{g r}, \quad(\cdot)=\frac{\mathrm{d}()}{\mathrm{d} s}=\frac{r}{V} \frac{\mathrm{d}()}{\mathrm{d} t} .
$$

For constant flight altitude, the lift coefficient is related to the bank angle by the relation:

$$
\frac{C_{L}}{C_{L}^{*}}=\frac{\omega(1-v)}{v \cos \sigma} .
$$

The initial speed is taken as slightly subcircular. Hence, we have the initial condition:

$$
s_{0}=0, \quad \theta_{0}=\phi_{0}=\psi_{0}=0, \quad v_{0}=0.95 .
$$

At the final time, for a closed circuit glide, we have:

$$
s_{f}=\text { free }, \quad \theta_{f}=\phi_{f}=0, \quad \psi_{f}=\text { free }, \quad v=v_{f} .
$$

The final speed is the stall speed, computed from eqn (43) with $C_{L}=C_{L_{\max }}, \sigma=0$. In the computation, we take the value $\omega=1 / 3, C_{L_{\max }} / C_{L}^{*}=2.2$ and this results in $v_{f}=1 / 7.6$. Below this speed, level flight can no longer be maintained at the altitude selected.

At any instant, the angle $\delta$ from the vehicle to the origin is given by $\cos \delta=\cos \theta \cos \phi$. Hence, we maximize the maximum of the function

$$
F=-\cos \theta \cos \phi
$$

over the interval $\left[s_{0}, s_{f}\right]$.

First we notice that

$$
\dot{F}=\sin \theta \cos \psi+\cos \theta \sin \phi \sin \psi .
$$

We have a $q>1$ problem. Again, we can conclude by inspecting the domain of maneuverability that the 
bank control is continuous at the jump point where $\dot{F}\left(s_{1}\right)=0$. This also can be seen by forming the Hamiltonian

$$
\begin{aligned}
H= & p_{\theta} \frac{\cos \psi}{\cos \phi}+p_{\phi} \sin \psi-p_{v} \frac{v}{E^{*} \omega} \\
& \times\left[1+\frac{\omega^{2}(1-v)^{2}}{v^{2}}\left(1+\tan ^{2} \sigma\right)\right] \\
& +p_{\psi}\left[\frac{(1-v)}{v} \tan \sigma-\cos \psi \tan \phi\right]
\end{aligned}
$$

and maximizing it with respect to the bank control to have the optimum condition

$$
\tan \sigma=\frac{E^{*} p_{\psi}}{2 \omega(1-v) p_{v}} .
$$

Then, since $v$ and $\psi$ are not contained in the function $F$, their adjoints are continuous at the jump point and by eqn (49) the bank angle is continuous at the point.

Again, in this problem, we have the classical integrals[8]

$$
\begin{aligned}
& H=C_{0}, \quad p_{\theta}=C_{1} \\
& p_{\phi}=C_{2} \sin \theta-C_{3} \cos \theta \\
& p_{\psi}=C_{1} \sin \phi+\left(C_{2} \cos \theta+C_{3} \sin \theta\right) \cos \phi .
\end{aligned}
$$

First, we notice that for a time free problem $C_{0}^{+}=C_{0}^{-}=0$. Then, for a maximax problem with one single isolated maxima, $\mu=-1$, we use the jump condition (11) to obtain

$$
\begin{aligned}
& C_{1}^{+}=C_{1}^{-}-\sin \theta_{1} \cos \phi_{1} \\
& \begin{aligned}
C_{2}^{+} & \sin \theta_{1}-C_{3}^{+} \cos \theta_{1} \\
& =C_{2}^{-} \sin \theta_{1}-C_{3}^{-} \cos \theta_{1}-\cos \theta_{1} \sin \phi_{1}
\end{aligned} \\
& \begin{aligned}
C_{1}^{+} & \sin \phi_{1}+\left(C_{2}^{+} \cos \theta_{1}+C_{3}^{+} \sin \theta_{1}\right) \cos \phi_{1} \\
& =C_{1}^{-} \sin \phi_{1}+\left(C_{2}^{-} \cos \theta_{1}+C_{3}^{-} \sin \theta_{1}\right) \cos \phi_{1} .
\end{aligned}
\end{aligned}
$$

Using the first equation, we rewrite the other two equations

$$
\begin{aligned}
\left(C_{2}^{+}-C_{2}^{-}\right) \cot \theta_{1}+\left(C_{3}^{+}-C_{3}^{-}\right) & =\sin \phi_{1} \\
-\left(C_{2}^{+}-C_{2}^{-}\right) \tan \theta_{1}+\left(C_{3}^{+}-C_{3}^{-}\right) & =\sin \phi_{1} .
\end{aligned}
$$

We deduce that $C_{2}^{-}=C_{2}^{+}=p_{\psi}\left(s_{f}\right)=0$. Hence, we have the jump relation:

$$
C_{1}^{+}=C_{1}^{-}-\sin \theta_{1} \cos \phi_{1}, \quad C_{3}^{+}=C_{3}^{-}+\sin \phi_{1} .
$$

As before, we select $C_{1}^{-}$and $C_{3}^{-}$with the transformation (53) to match the prescribed end conditions while using the optimal law

$$
\tan \sigma=\frac{-B+\sqrt{B^{2}+\left(p_{\psi} / \omega\right)^{2}\left[1+\left(\omega^{2} / v^{2}\right)(1-v)^{2}\right]}}{p_{\psi}(1-v) / v}
$$

where

$$
p_{\psi}=C_{1} \sin \phi+C_{3} \sin \theta \cos \phi
$$

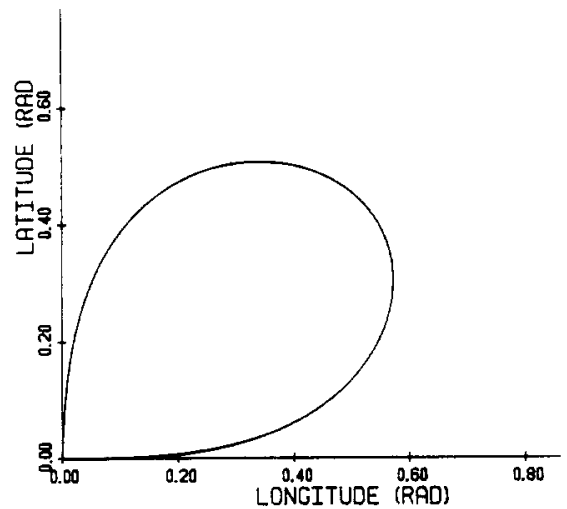

Fig. 6. Maximum flight radius over a spherical earth.

and

$$
\begin{aligned}
B=C_{1} & \cos \phi \cos \psi \\
& -C_{3}(\cos \theta \sin \psi+\sin \theta \sin \phi \cos \psi) .
\end{aligned}
$$

At the jump point, the bank angle is continuous but its derivative is discontinuous according to

$$
\frac{\dot{\sigma}^{-}-\dot{\sigma}^{+}}{\sin 2 \sigma}=\frac{1}{2 p_{\psi}} \sqrt{1-\cos ^{2} \theta_{1} \cos ^{2} \phi_{1}}=\frac{\sin \delta_{1}}{2 p_{\psi}},
$$

where $\delta_{1}$ is the maximized range angle.

Figure 6 shows the trajectory computed with the given data while Fig. 7 presents the history of the optimal bank angle. A sight discontinuity in the slope is observed at the point of maximum range. The value of $E^{*}$ used for the computation is rather high for any hypervelocity vehicle in the near future. We have experimented with lower values of $E^{*}$ but since we have restricted the flight at constant high altitude, a closed circuit flight is not achievable before the stall speed. On the other hand, with low value of maximum lift-to-drag ratio, closed circuit glide is feasible if we allow the altitude to decrease to sea level, or if we add a thrust to partially cancel the drag.

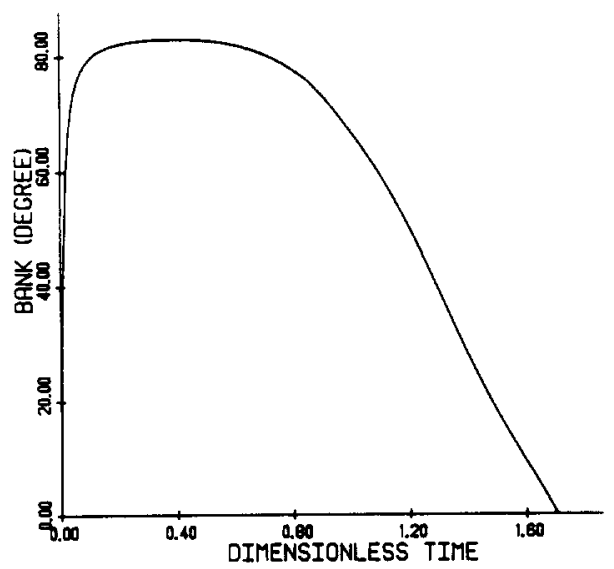

Fig. 7. Bank control for maximum flight radius. 


\section{A MAXIMAX PROBLEM WITH $q=1$}

It has been mentioned that for the case $q=1$ with isolated maxima, the control is in general discontinuous in the maximax problem. We consider the following problem:

$$
\begin{aligned}
& \max _{t \in\left[0, t_{f}\right]}\left(\max x_{2}\right) \\
& \dot{x}_{1}=x_{2}+u^{2} \\
& \dot{x}_{2}=-x_{2}+u
\end{aligned}
$$

$x_{1}(0)$ and $x_{1}\left(t_{f}\right)$ given with $t_{f}$ prescribed.

$$
x_{2}(0)=x_{2}\left(t_{f}\right)=0 \text {. }
$$

We introduce a component $x_{3}$ to be maximized at the final time, subject to

$$
\dot{x}_{3}=0, x_{3}(0)=\text { free, } x_{2}-x_{2} \leq 0 \text {. }
$$

The Hamiltonian of the problem is

$$
H=p_{1}\left(x_{2}+u^{2}\right)+p_{2}\left(-x_{2}+u\right),
$$

with solution for the adjoint

$$
p_{1}=C_{1}, p_{2}=C_{2} \mathrm{e}^{t}+C_{1},
$$

and optimal control

$$
u=-\frac{p_{2}}{2 p_{1}}=-\frac{1}{2}\left(\frac{C_{2}}{C_{1}} \mathrm{e}^{t}+1\right) .
$$

Here $F=x_{2}$ and with one maximum, $\mu_{1}=-1$, the jump condition (11) provides:

$$
p_{1}^{+}=p_{1}^{-}, \quad p_{2}^{+}=p_{2}^{-}-1 .
$$

Then, at the time $t_{1}$

$$
C_{1}^{+}=C_{1}^{-}, \quad C_{2}^{+}=C_{2}^{-}-\mathrm{e}^{-r_{1}} .
$$

The discontinuity in the control is seen from

$$
u^{+}-u^{-}=-\frac{1}{2 C_{1}}\left(C_{2}^{+}-C_{2}^{-}\right) \mathrm{e}^{t_{1}}=\frac{1}{2 C_{1}} .
$$

The problem is completely integrable. Let

$$
C_{2}^{-}=k C_{1}^{-}=k C_{1}^{+}=k C \text {. }
$$

Enforcing only the terminal conditions $x_{2}(0)=x_{2}\left(t_{f}\right)=0$ while leaving $x_{1}(0)$ and $x_{1}\left(t_{f}\right)$ as specified parameters, we have the solution in the interval $\left[0, t_{1}\right]$ :

$$
\begin{aligned}
x_{2}(t)= & \left(\frac{k}{4}+\frac{1}{2}\right) \mathrm{e}^{-t}-\frac{k}{4} \mathrm{e}^{t}-\frac{1}{2} \\
x_{1}(t)= & -\frac{t}{4}+\frac{k}{4} \mathrm{e}^{t}-\left(\frac{k}{4}+\frac{1}{2}\right) \mathrm{e}^{-t} \\
& +\frac{k^{2}}{8} \mathrm{e}^{2 t}+\frac{1}{2}-\frac{k^{2}}{8}+x_{1}(0) .
\end{aligned}
$$

After the time $t_{1}$, with the jump relation (64) we have the control:

$$
u(t)=-\frac{1}{2 C}\left(k C-\mathrm{e}^{-t_{1}}\right) \mathrm{e}^{t}-\frac{1}{2} .
$$

By integrating the state equations starting from $x_{1}\left(t_{1}\right)$ and $x_{2}\left(t_{1}\right)$, we have the trajectory for the second half

$$
\begin{aligned}
x_{2}(t)= & \left(\frac{k}{4}+\frac{1}{2}-\frac{\mathrm{e}^{t_{1}}}{4 C}\right) \mathrm{e}^{-t}-\frac{1}{4 C}\left(k C-\mathrm{e}^{-t_{1}}\right) \mathrm{e}^{t}-\frac{1}{2} \\
x_{1}(t)= & -\frac{t}{4}+\frac{1}{4 C}\left(k C-\mathrm{e}^{-t_{1}}\right) \mathrm{e}^{t} \\
& -\left(\frac{k}{4}+\frac{1}{2}-\frac{1}{4 C} \mathrm{e}^{t_{1}} e^{-t}\right)+\frac{1}{8 C^{2}}\left(k C-\mathrm{e}^{-t_{1}}\right)^{2} \mathrm{e}^{2 t} \\
& +\frac{1}{2}-\frac{k^{2}}{8}-\frac{1}{8 C^{2}}+\frac{k}{4 C} \mathrm{e}^{t_{1}}+x_{1}(0)
\end{aligned}
$$

We notice that the trajectory $x_{2}(t)$ is symmetric with respect to the line $t_{1}$ since $x_{2}^{-}(t)=x_{2}^{+}\left(2 t_{1}-t\right)$ if

$$
k \mathrm{e}^{2 t_{1}}+(k+2)-\frac{1}{C} \mathrm{e}^{t_{1}}=0 .
$$

But, this is the same as when we write the condition that $x_{2}\left(2 t_{1}\right)=x_{2}(0)=0$. Therefore, if we use the initial condition $x_{2}(0)=0$, the trajectory $x_{2}(t)$ is always symmetric in the interval $\left[0,2 t_{1}\right]$ with respect to $t_{1}$ and if we furthermore impose the condition $x_{2}\left(t_{f}\right)=0$, we have the exact solution:

$$
t_{1}=t_{f} / 2
$$

when $x_{2}$ is maximized. By writing the condition that $x_{1}\left(t_{f}\right)=x_{1}\left(2 t_{1}\right)$ and using eqn (70) for simplification, we have

$$
x_{1}(0)-x_{1}\left(t_{f}\right)+\frac{1}{2}+\frac{k}{2}-\frac{t_{1}}{2}-\frac{1}{8 C^{2}}+\frac{k}{4 C} \mathrm{e}^{t_{1}}=0 .
$$

Finally by eliminating $C$ between the eqns (70) and (72), we have a quadratic equation for $k$.

$$
\begin{aligned}
\frac{1}{8}\left(\mathrm{e}^{2 t_{1}}-\mathrm{e}^{-2 t_{1}}\right) k^{2} & +\frac{1}{2}\left(1-\mathrm{e}^{-2 t_{1}}\right) k+x_{1}(0) \\
& -x_{1}\left(t_{f}\right)+\frac{1}{2}-\frac{t_{1}}{2}-\frac{1}{2} \mathrm{e}^{-2 t_{1}}=0 .
\end{aligned}
$$

The condition for real roots is

$$
t_{1}-\tanh t_{1} \geq 2\left[x_{1}(0)-x_{1}\left(t_{f}\right)\right] .
$$

The equation is general, subject only to the condition that $x_{2}(0)=x_{2}\left(t_{f}\right)=0$. For the case where $t_{f}=3$, we have $t_{1}=1.5$, and taking $x_{1}(0)=-5, x_{1}\left(t_{f}\right)=0$, we have:

$$
\begin{aligned}
k & =-1.549221 \\
C_{1}^{-} & =-0.146144 \\
C_{2}^{-} & =0.226410 .
\end{aligned}
$$

The maximized value of the maximum of $x_{2}$ is

$$
x_{2}\left(t_{1}\right)=1.260927 \text {. }
$$

Figure 8 shows the variation of $x_{2}(t)$ and Fig. 9 shows the variation of the optimum control $u(t)$ with discontinuity at $t_{1}$. For comparison, we have plotted in Fig. 8 in dashed line the trajectory $x_{2}(t)$ with the maximum enclosed area, giving a maximum value of $x_{2}$ equal to 0.9256 . 


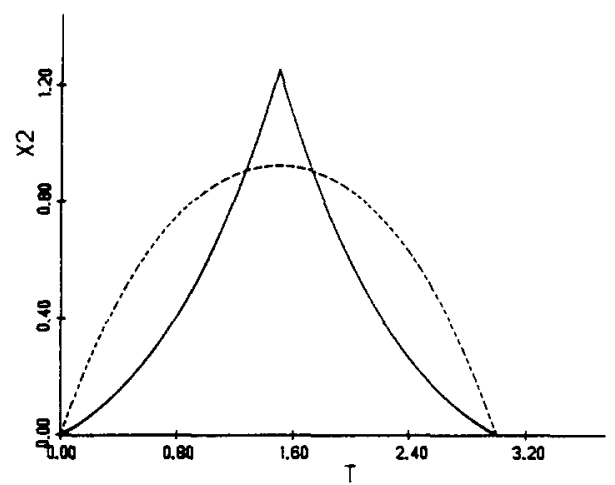

Fig. 8. Optimal trajectory for $\max \left(\max x_{2}\right)$.

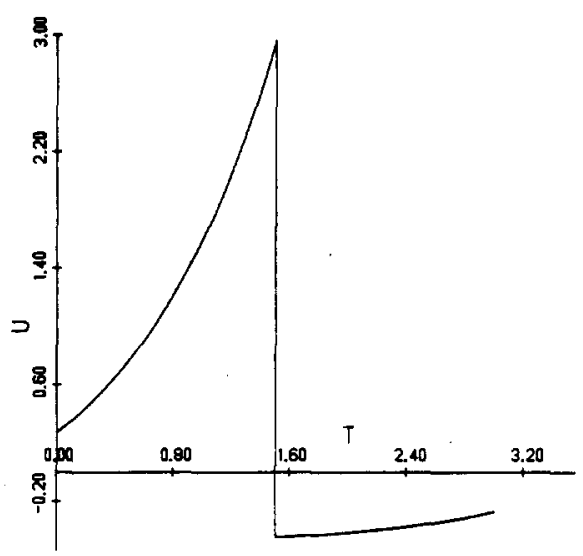

Fig. 9. Optimal control with discontinuity at $t_{1}$.

\section{CONCLUSION}

The necessary conditions for the minimax (or maximax) problem have been derived by transforming the problem into an optimal control problem with state constraint. The jump conditions obtained are applicable to all problems with a single or multiple maxima. For problems with flat maxima, it is necessary that the first time derivative of the minimax function contains all the control variables or only one scalar control is involved. If the maxima are isolated points, the order of the derivative in which the control appears explicitly for the first time can be arbitrary. In particular, the continuity of the control at the jump point is discussed.

Several examples were considered to illustrate the application of the theory.

Acknowledgement-This work was supported by the Jet Propulsion Laboratory under contract No. 956416 with Dr K. D. Mease as project manager.

\section{REFERENCES}

1. C. D. Johnson, Optimal control with Chebyshev minimax performance index. ASME Trans. Basic Enging 89, 251-262 (1967).

2. W. F. Powers, A Chebyshev minimax technique orientated to aerospace trajectory optimization problems. AIAA J. 10, 1291-1296 (1972).

3. L. S. Pontryagin et al., The Mathematical Theory of Optimal Processes, Chap. 6. Interscience Publishers, New York (1962).

4. P. Contensou, Conditions d'optimalité pour les domaines de manoeuvrabilité à frontière semi-affine. Lecture Notes in Mathematics, No. 112, Springer-Verlag (1970)

5. N. X. Vinh, Optimal singular control with applications to trajectory optimization. NASA CR-3087 (1979).

6. A. E. Bryson and Y. C. Ho, Applied Optimal Control, Chaps 3 and 9. Blaisdell, Waltham, Mass. (1969).

7. N. X. Vinh, C. Y. Yang and J. S. Chern, Optimal trajectories for maximum endurance gliding in a horizontal plane. AIAA J. Guidance 7, 246-248 (1984).

8. N. X. Vinh, Optimal Trajectories in Atmospheric Flight, Chap. 12. Elsevier, Amsterdam (1981). 